\title{
Effective Dose of CT-Guided Epidural and Periradicular Injections of the Lumbar Spine: A Retrospective Study
}

\author{
Juraj Artner*, Friederike Lattig, Heiko Reichel and Balkan Cakir
}

\author{
Department of Orthopaedic Surgery, University of Ulm, RKU, Germany
}

\begin{abstract}
Spinal injection procedures can be performed blindly or, more accurately, with fluoroscopic or computed tomography (CT) guidance. Radiographic guidance for selective nerve root blocks and epidural injections allows an accurate needle placement, reduces the procedure time and is more secure for the patient, especially in patients with marked degenerative changes and scoliosis, resulting in a narrowing of the interlaminar space. Limiting factors remain the availability of scanners and the radiation dose. Interventional CT scan protocols in axial CT-acquisition mode for epidural and periradicular injections help to limit the radiation dose without a significant decrease of image quality. The purpose of this retrospective study was to analyze the effective radiation dosage patients are exposed during CT-guided epidural lumbar and periradicular injections. A total amount of $n=1870$ datasets from 18 months were analyzed after multiplying the dose length product with conversion factor $\mathrm{k}$ for each lumbar segment. For lumbar epidural injections $(\mathrm{n}=1286)$, a mean effective dose of $1.34 \mathrm{mSv}$ (CI 95\%, 1.30-1.38), for periradicular injections $(\mathrm{n}=584)$ a mean effective dose of 1.38 $\mathrm{mSv}(\mathrm{CI} 95 \%, 1.32-1.44)$ were calculated.
\end{abstract}

Keywords: Computed tomography, CT, guidance, epidural, perineural, periradicular, injections, spinal, interventions, radiation, dose.

\section{INTRODUCTION}

Computed tomography (CT) is a well-accepted tool for the precise needle placement in many spinal interventions. The mean benefits are accuracy, reduction of complication rates and patient comfort due to the minimal invasive procedure. Despite these benefits, the radiation dose still remains a serious concern, contributing to a controversial discussion between blind punctions and radiographicguidance in Germany [1-3]. Because of the increasing amount of spinal disorders among the population, the need for conservative spinal interventions is increasing. In fact, due to reports of the Medicare population, the rate of epidural injections is rapidly growing [4].

\section{MATERIAL AND METHODS}

Datasets from 1870 CT-guided lumbar injections between 09.11.2009 and 09.05.2011 were analyzed for the radiation dose according to the type of procedure and segment, making a total amount of 1286 epidural lumbar injections and 584 lumbar nerve blocks (lumbar epidural injections $\mathrm{n}=932 / \mathrm{yr}$, lumbar nerve blocks $\mathrm{n}=387 / \mathrm{yr}$ ). For CTguided interventions we use the SOMATOM Emotion scanner (syngo CT 2009E, 16-slice solution, Siemens Medical Solutions AG, Erlangen, Germany) with interventional protocol (axial CT-acquisition, topogram: 120 $\mathrm{mAs}, 130 \mathrm{kV}$, scan: $80 \mathrm{mAs}, 130 \mathrm{kV}$, intervention: $50 \mathrm{mAs}$, $130 \mathrm{kV}$ ) to reduce the radiation exposure. The injection is provided by experienced orthopaedic surgeons, specialized in the conservative therapy of spinal disorders using the techniques described by Wagner $[5,6]$. A total amount of

*Address correspondence to this author at the Department of Orthopaedic Surgery, University of Ulm, RKU, Oberer Eselsberg 45, 89081 Ulm, Germany; Tel: 0049-731-177-5111; E-mail: j.artner@gmail.com
1870 patients undergoing CT-guided spinal injections were included into the retrospective data analysis study. Effective dose was calculated by multiplying dose-length product (DLP) and conversion factor ( $\mathrm{k}$ factor) $[7,8]$. For each lumbar segment, descriptive statistical analysis was performed with SPSS Statistics 17.0, IBM, Armonk, New York 10504, 2008.

\section{RESULTS}

A total amount of 1286 epidural lumbar injections and 584 lumbar nerve blocks were performed with CT-guidance. Per patient, there were 3-6 scans needed for the procedure, with an average intervention time of 5-10 minutes (positioning, unclothing, desinfection, preparation, topogramm, intervention). There were no complications during the interventions. For epidural lumbar injections $(n=1286)$, mean effective dose (E) of $1.34 \mathrm{mSv}$ (CI 95\%, $1.30-1.38)$ was calculated, for lumbar nerve blocks $(\mathrm{n}=584)$, mean effective dose was $1.38 \mathrm{mSv}$ (CI 95\%, 1.32-1.44) (see Fig. 1 and Table 1).

\section{DISCUSSION}

There is a controversy regarding the optimal imagingtechnique for spinal injection procedures in the international literature. While some authors prefer blind injections, there are also interventionalists using sonography-, MRI-, fluoroscopy- or CT-guidance for a safe epidural or periradicular needle placement [3, 9-12].

The CT-guided technique is described as fast, safe, and highly accurate in proving appropriate needle placement in epidural injections [8] (see Fig. 2). For periradicular injections, CT- guided injections are described to be superior to fluoroscopy-guided for both the visualization and a 


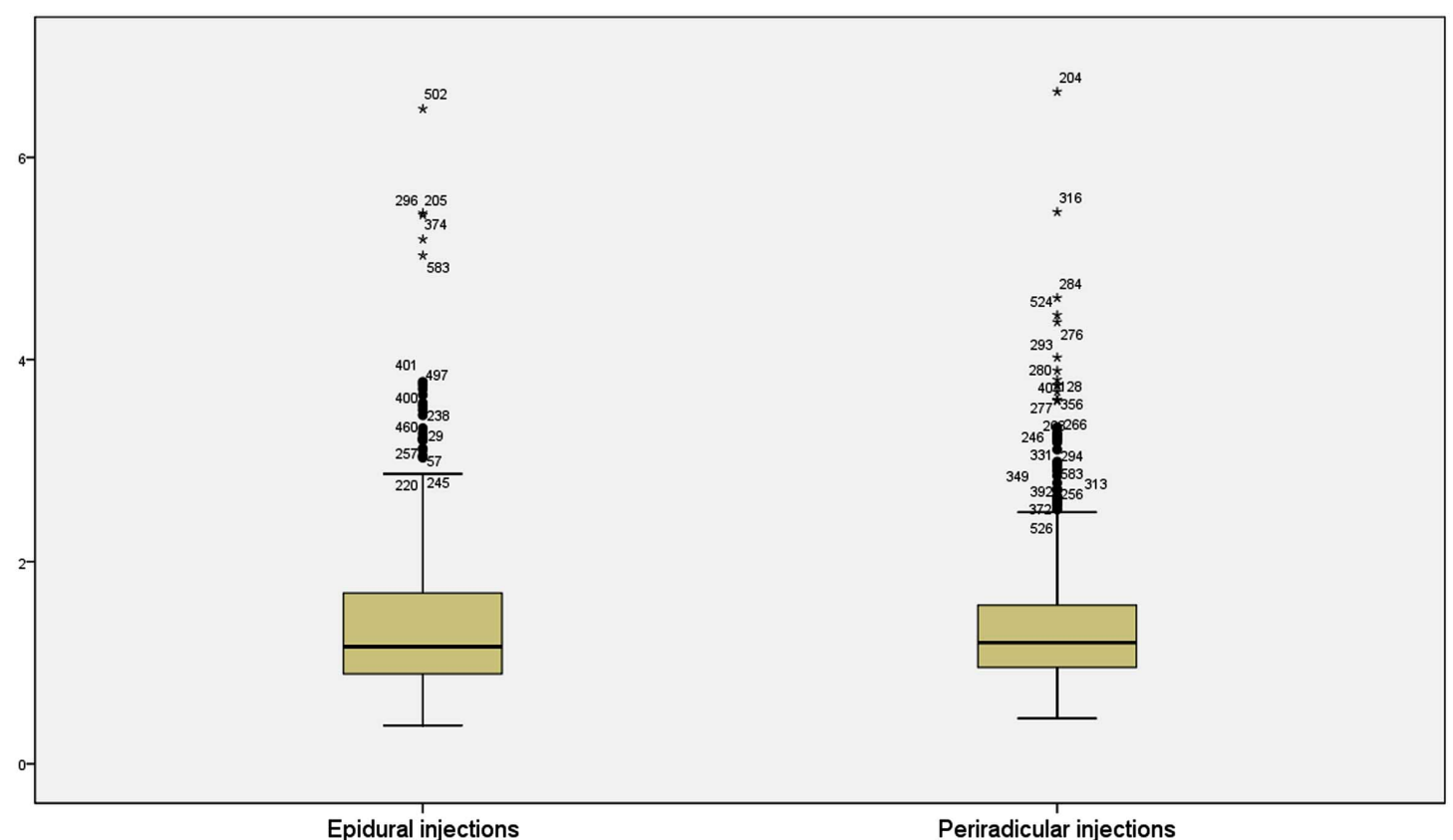

Fig. (1). Effective Dose in $\mathrm{mSv}$ in lumbar epidural (left) and periradicular (right) injections.
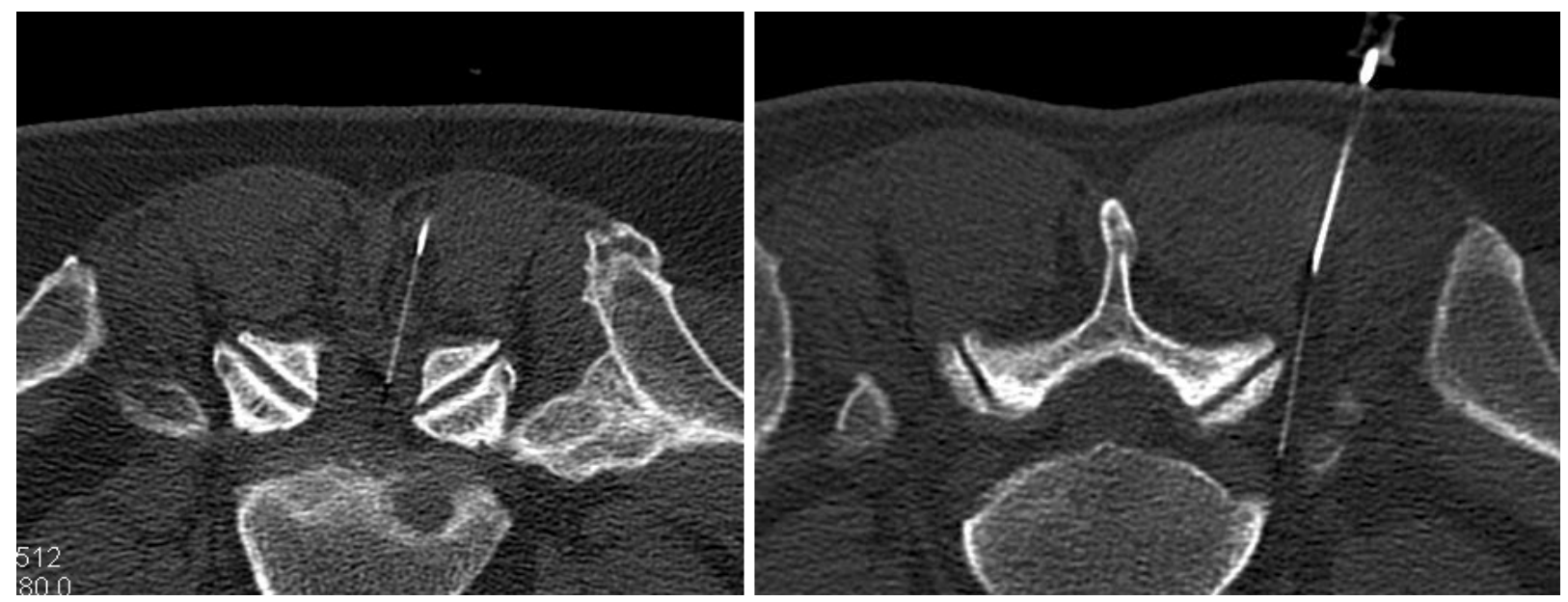

Fig. (2). CT-guided epidural injection at the level L5/S1 (left image) and periradicular injection near the L5-nerve root (right image) in the standard interventional protocol (axial CT-acquisition).

Table 1. Segmental Distribution of Effective Doses in Epidural und Periradicular Lumbar Injections

\begin{tabular}{|c|c|c|c|c|c|}
\hline Procedure & Number of Injections n & Mean Effective Dose mSv & Confidence Interval 95\% & Median eff. Dose mSv & Standard Deviation \\
\hline \hline EDU L 1/2 & 9 & 1.04 & $0.62-1.46$ & 0.87 & 0.55 \\
\hline EDU L 2/3 & 142 & 1.31 & $1.21-1.40$ & 1.14 & 0.57 \\
\hline EDU L 3/4 & 295 & 1.36 & $1.28-1.45$ & 1.11 & 0.74 \\
\hline EDU L 4/5 & 563 & 1.37 & $1.30-1.44$ & 1.13 & 0.80 \\
\hline EDU L 5/s1 & 277 & 1.29 & $1.21-1.38$ & 1.17 & 0.70 \\
\hline Radix L1 & 0 & - & -1.14 & - \\
\hline Radix L2 & 22 & 1.25 & $1.11-1.48$ & 1.17 \\
\hline Radix L3 & 44 & 1.3 & $1.18-1.36$ & 1.22 & 0.46 \\
\hline Radix L4 & 131 & 1.27 & $1.37-1.54$ & 1.11 & 0.50 \\
\hline Radix L5 & 322 & 1.46 & $1.17-1.50$ & 0.81 \\
\hline Radix S1 & 65 & 1.33 & & \\
\hline
\end{tabular}


longer-lasting effect [10]. Despite the accuracy, the patient's and physician's exposure to radiation in CT -guided interventions remains a serious concern [13].

There are two possible types of radiation effects on human body: the so called deterministic effects above a threshold dosage (like erythema, tissue necrosis, epilation, hematopoietic or gastrointestinal damage) and stochastic effects (like genetic effects and cancer induction), which do not have a threshold dose [14]. Clinical symptoms of radiation exposure start to appear at doses more than $0.5 \mathrm{~Gy}$. The most radiosensitive tissues are haematopoietic and lymphatic systems, responding already to single exposures as small as $0.5-1$ Gy. Mitotic cell division is delayed in doses greater than $0.5 \mathrm{~Gy}$ and at more than $3 \mathrm{~Gy}$, the mitotic rate is irreversibly arrested [15]. Radiation dermatitis is induced above the $2000 \mathrm{mGy}$ threshold, while radiation-induced skin changes were also reported at levels of $1000 \mathrm{mGy}$ [16]. The physicians should be concerned about both effects in interventions using ionizing radiation. While working under the threshold doses of deterministic effects prevents these damages, the cumulative stochastic effects of repetitive exposure to low dose radiation are random, may take decades to manifest and can only be simulated. Cancer induction is the most important stochastic effect, estimated at absorbed doses of less than $1 \mathrm{~Gy}$ [17]. Leukemia seems to be associated with chronic radiation exposures with doses as low as 50-100 cGy, with a linear correlation between dose and incidence in doses between 100-500 cGy [15]. Other stochastic effects associated with exposure to ionizing radiation are thyroid, bone, lung and other cancers.

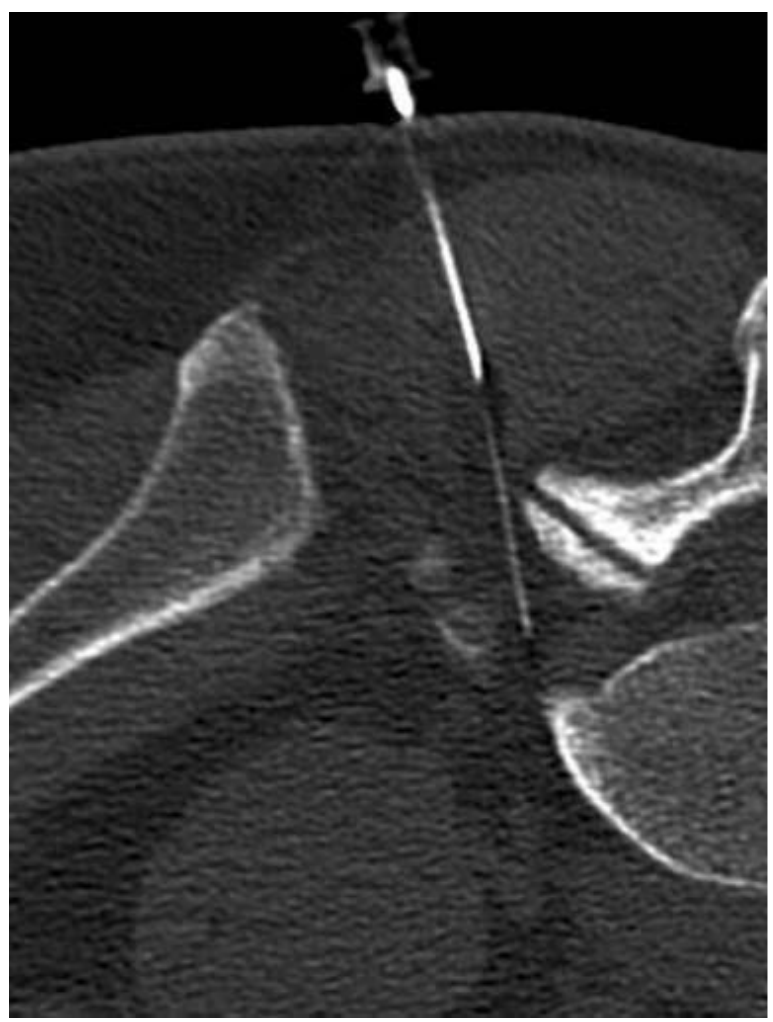

There is an amount of radiation exposition doses in spinal CT- examinations and interventions described in literature, which vary significantly between and within institutions, depending on the settings, the interventionalist and the preferred technique $[18,19]$. Leng et al. compared the effective doses using the k- conversion factor for the helical mode and Monte Carlo organ dose coefficients for intermittent mode for interventional CT procedures [20]. For injections, they described an effective dose of $9.1 \pm 5.5 \mathrm{mSv}$. Carlson et al. described a median overall patient radiation dose of $738 \mathrm{mGy}$ (corresponding to approx. $11.07 \mathrm{mSv}$ ) for CT-guided procedures like aspirations, biopsies and catheter drainages [21]. Shepherd et al. reported a mean total DLP of $199 \pm 101 \mathrm{mGy}$ for spinal injection procedures after changing CT acquisitions from helical to axial mode [22], corresponding to a mean effective radiation dose of 2.99 $\mathrm{mSv}$ (for lumbar injections only=3.3 mSv). Schmid et al. compared the radiation doses in CT- and fluoroscopy-guided epidural injections using an Alderson Rando phantom at the lumbar segment L4/5 [23]. They described effective doses of 1.51 to $3.53 \mathrm{mSv}$ (4-10 scans) for CT-guidance for standard protocols and 0.22 to $0.43 \mathrm{mSv}$ (4-10 scans) for low dose protocols. For fluoroscopic guidance they described effective doses of 0.43 to $1.25 \mathrm{mSv}(1-3 \mathrm{~min})$ in continuous mode and less than $0.1 \mathrm{mSv}$ for pulsed mode (1min, 3 pulses $/ \mathrm{sec}$ ). Hoang et al. calculated mean radiation doses of $0.85 \mathrm{mSv}$ for lumbar epidural steroid injections using conventional fluroscopy-guidance and $3.35 \mathrm{mSv}$ for CT-fluoroscopy guidance, when a full diagnostic lumbar scan was performed as a part of the procedure [24]. Depending on the tube current and energy settings there are also reports of CTguided interventions, which lead to much higher radiation

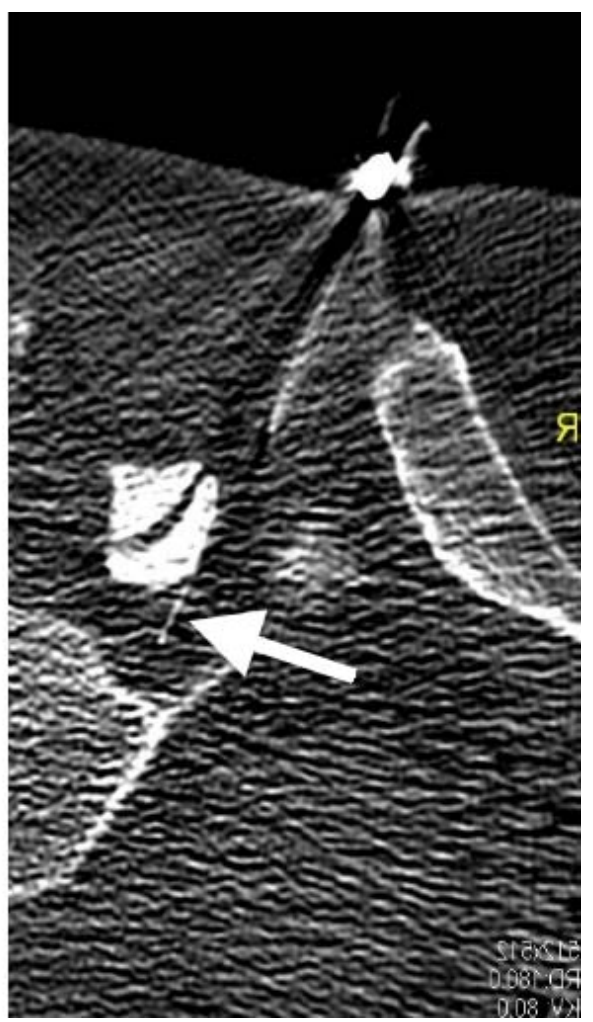

Fig. (3). Comparison of standard interventional protocol (left image) and low-dose protocol (right image) in CT-guided L5-nerve root block. Despite the reduced contrast in the low-dose image, the needle (29 gauge, most thin needle caliber) is still visible as good as the bony landmarks of the neuroforamen. A dose reduction of $85 \%$ was achieved. 
exposures, in some cases up to $830 \mathrm{mGy}$ per procedure (corresponding to $12.45 \mathrm{mSv}$ ) [25]. Our measures correspond to radiation doses in Schmid 's study for standard interventional CT-protocols. Despite the fact that these doses are lower than doses mentioned in other studies and below the threshold of deterministic radiation effects mentioned above, one must be concerned about the cumulative stochastic effects of repetitive injection therapy.

To determine the risk of cancer induction in computed tomography examinations and interventions is not unproblematic. Based on phantom simulations for spinal computed tomography, Richards et al. calculated a relative risk ratio for inducing a cancer of 1:3200 for diagnostic scans of the whole lumbar spine in standard diagnostic protocol and 1:200000 for low dose protocol with an estimated effective dose of $0.1 \mathrm{mSv}$. Narrowing the area of interest to dorsal structures from L3-L5 (effective dose of 3.5 $\mathrm{mSv}$ ) in standard protocol also reduced the risk to $1: 5200$ [26].

The cumulative radiation exposure of patients with spinal disorders undergoing CT examinations and interventions is underestimated. Despite the good image quality and efficacy of CT-guided lumbar procedures, the radiation dose reduction should be targeted in further studies. There is a wide range of possibilities how to minimize the radiation dose exposures in CT-guided interventions. Switching from helical to axial image acquisition, reducing the tube current, depending on patient 'body habitus (AP diameter), tailoring the planning CT scan, narrowing the area of interest, as good as switching to CT-fluoroscopic navigation and reducing the scatter exposure (use of lead drape and radiation protection gloves) are described in literature [13, 22, 23]. Since 2011 we develop in our department low dose protocols which help to reduce the radiation exposure with an acceptable image quality according to the ALARA-principle (as low as reasonably achievable). Fig. (3) demonstrates an example of CT-guided periradicular injection comparing standard interventional protocol and low-dose protocol, where a dose reduction of $85 \%$ could be achieved (see Fig. 3).

\section{CONCLUSION}

The radiation exposure in CT-guided spinal injections is underestimated. Because of the cumulative effect of repetitive spinal injections under CT-guidance in patients with spinal disorders and the large variety of used radiation doses between the performing interventionalists a reduction of radiation dose and standardization of CT-protocols should be targeted in further studies.

\section{ACKNOWLEDGEMENT}

Declared none.

\section{CONFLICT OF INTEREST}

The authors confirm that this article content has no conflicts of interest.

\section{ABBREVIATIONS}

$$
\begin{array}{ll}
\mathrm{CT} & =\text { Computed tomography } \\
\mathrm{DLP} & =\text { Dose length product } \\
\mathrm{Gy} & =\text { Gray }
\end{array}
$$

$\begin{array}{ll}\mathrm{Sv} & =\text { Sievert } \\ \mathrm{CI} & =\text { Confidence interval } \\ \mathrm{DLP} & =\text { Dose length product } \\ \mathrm{EDU} & =\text { Epidural injection (to determine segment) } \\ \text { Radix } & =\text { Nerve root } \\ \mathrm{mAs} & =\text { Milliampereseconds } \\ \mathrm{kV} & =\text { Kilovolt }\end{array}$

\section{REFERENCES}

[1] Krämer J, Blettner M, Hammer GP. Image-guided injection therapy in the lumbar spine. Dtsch Arztebl 2008; 105(34-35): 596-8.

[2] Lutze MA, Morley T, Großkurth D, Krämer J. Diskussion: Schmerztherapie an der Wirbelsäule. Ist CT-gesteuerte Injektion notwendig? Dtsch Arztebl 1998; 95(38): A2333-4.

[3] Theodoridis T, Krämer J. Spinal injection techniques. New York: Thieme 2009.

[4] Friedly J, Chan L, Deyo R. Increases in lumbosacral injections in the Medicare population: 1994-2001. Spine 2007; 32: 1754-60.

[5] Wagner AL. CT Fluoroscopy-guided epidural injections: Technique and results. AJNR Am J Neuroradiol 2004; 25: 1821-3.

[6] Wagner AL. Selective lumbar nerve root blocks with CT fluoroscopic guidance: technique, results, procedure time, and radiation dose. AJNR Am J Neuroradiol 2004; 25: 1592-4.

[7] Shrimpton P. Assessment of patient dose in CT. In: EUR. European guidelines for multislice computed tomography funded by the European Commission 2004: contract number FIGMCT200020078-CT-TIP. Luxembourg, Luxembourg: European Commission. Appendix C14, 2004.

[8] Shrimpton PC, Hillier MC, Lewis MA, Dunn M. Doses from computed tomography (CT) examinations in the UK: 2003 review. Report NRPB-W67 .Chilton, UK: National Radiological Protection Board 2005.

[9] Silbergleit R, Mehta BA, Sanders WP, Talati SJ. Imaging-guided injection techniques with fluoroscopy and $\mathrm{CT}$ for spinal pain management. Radiographics 2001; 21(4): 927-39.

[10] Lutze M, Stendel R, Vesper J, Brock M. Periradicular therapy in lumbar radicular syndromes: methodology and results. Acta Neurochir 1997; 139(8): 719-24.

[11] Loizides A, Peer S, Plaikner M, et al. Ultrasound-guided injections in the lumbar spine. Med Ultrason 2011; 13(1): 54-8.

[12] Fritz J, Thomas Ch, Clasen S, Claussen CD, Lewin JS, Pereira PL. Freehand real-time MRI-guided lumbar spinal injection procedures at $1.5 \mathrm{~T}$ : Feasibility, accuracy, and safety. AJR Am J Roentgenol 2009; 192: 161-7.

[13] Stoeckelhuber BM, Schulz E, Melchert UH, et al. Procedures, spectrum and radiation exposure in CT-fluoroscopy. Rontgenpraxis 2003; 55(2): 51-7.

[14] Kamiya K, Sasatani M. Effects of radiation exposure on human body. Nihon Rinsho 2012; 70(3): 367-74.

[15] Bolus NE. Basic review of radiation biology and terminology. J Nucl Med Technol 2001; 29: 67-73.

[16] Paulson EK, Sheafor DH, Enterline DS, Mc Adams HP, Yoshizumi TT. CT Fluoroscopy-guided interventional procedures: techniques and radiation dose to radiologists. Radiology 2001; 220: 161-7.

[17] Mettler FA. Medical effects and risks of exposure to ionising radiation. J Radiol Prot 2012; 32: N9-13.

[18] Schmith-Bindman R, Lipson J, Marcus R, et al. Radiation dose associated with common computed tomography examinations and the associated lifetime attributable risk of cancer. Arch Intern Med 2009; 169(22): 2078-86.

[19] Schauberger JS, Kranz PG, Roy Choudhury K, Eastwood JD, Gray L, Hoang JK. CT-guided lumbar nerve root injections: are we using the correct radiation dose settings? AJNR Am J Neuroradiol 2012; [Epub ahead of print].

[20] Leng S, Christner JA, Carlson SK, et al. Radiation dose levels for interventional CT procedures. AJR Am J Roentgenol 2011; 197(1): w97-104.

[21] Carlson SK, Bender CE, Classic KL, et al. Benefits and safety of $\mathrm{CT}$ fluoroscopy in interventional radiologic procedures. Radiology 2001; 219: 515-20. 
[22] Shepherd TM, Hess CP, Chin CT, Gould R, Dillon WP. Reducing patient radiation dose during ct- guided procedures: demonstration in spinal injections for pain. AJNR Am J Neuroradiol 2011; 32(10): 1776-82.

[23] Schmid G, Schmitz A, Borchardt D, et al. Effective dose of CTand fluoroscopy- guided perineural/epidural injections of the lumbar spine: a comparative study. Cardiovase Intervent Radiol 2006; 29(1): 84-91.
[24] Hoang JK, Yoshizumi TT, Toncheva G, et al. Radiation dose exposure for lumbar spine epidural steroid injections: a comparison of conventional fluoroscopy data and ct fluoroscopy techniques. AJR Am J Roentgenol 2011; 197(4): 778-82.

[25] Nawfel RD, Judy PF, Silverman SG, Hooton S, Tuncali K, Adams DF. Patient and personnel exposure during CT-guided interventional procedures. Radiology 2000; 216: 180-4.

[26] Richards PJ, George J, Metelko M, Brown M. Spine computed tomography doses and cancer induction. Spine 2010; 35(4): 430-3.

Received: March 7, 2012

Revised: July 17, 2012

Accepted: July 22, 2012

(C) Artner et al.; Licensee Bentham Open.

This is an open access article licensed under the terms of the Creative Commons Attribution Non-Commercial License (http://creativecommons.org/licenses/by-nc/3.0/) which permits unrestricted, non-commercial use, distribution and reproduction in any medium, provided the work is properly cited. 